\title{
Can the Bone Mineral Density of the Non-fractured Contralateral Hip Predict Medial Migration of Helical Blade-type Lag Screws in Osteoporotic Hip Fractures?
}

\section{Suenghwan Jo}

Chosen University Hospital

Hyun Jun Lee

Chosen University Hospital

\section{Sang Hong Lee}

Chosun university

Je Hong Ryu

Chosen University

\section{Ba Rom Kim}

Chosen University

Dong Seop Lim

Chosun University

Soo Ah Kim ( $\sim$ ksaobgyn@gmail.com )

Chosun University, School of Medicine 365 Pilmundae-ro, Dong-gu, Gwangju 61453, South Korea https://orcid.org/0000-0002-9049-1741

Research article

Keywords: bone mineral density (BMD), contralateral hip, biomechanical tests, dual-energy X-ray

Posted Date: October 22nd, 2020

DOI: https://doi.org/10.21203/rs.3.rs-94889/v1

License: (c) (1) This work is licensed under a Creative Commons Attribution 4.0 International License. Read Full License 


\section{Abstract}

Background: In this study, we performed a biomechanical tests on the osteoporotic femoral heads to validate whether the bone mineral density (BMD) measured at the non-fractured contralateral hip can predict the BMD and potential medial migration of helical blade-type lag screws on the fractured femoral head.

Methods: Twenty-four osteoporotic femoral heads were retrieved from patients with femoral neck fracture undergoing hip arthroplasty. The patients had their BMD measured from the contralateral hip using dualenergy X-ray absorptiometry prior to surgery. The BMD of the retrieved femoral heads was measured using micro-computed tomography. The mechanical properties for the medial migration of lag screw were measured by performing an uniaxial compression test on the femoral head using a helical bladetype lag screw. Statistical analysis was performed to determine the correlation among the BMDs of the non-fractured contralateral hip and the retrieved femoral head and the mechanical properties of the fractured femoral head.

Results: The mean BMD of the contralateral hip was $0.60 \pm 0.14 \mathrm{~g} / \mathrm{cm}^{2}$ in the neck region and $0.63 \pm 0.15$ $\mathrm{g} / \mathrm{cm}^{2}$ in the total proximal femur. The mean BMD of the fractured femoral head was $463.3 \pm 48.05 \mathrm{mg}$ $\mathrm{HA} / \mathrm{cm}^{3}$. The mechanical properties of the fractured femoral head were measured to be $0.92 \mathrm{kN}$ for the maximum compressive load and $14.50 \pm 5.48 \mathrm{kN}$ for the accumulated compression load. In the analysis of the correlation among the parameters, we found no correlation between the BMD of the non-fractured contralateral hip and the mechanical properties of the fractured femoral head. In addition, we observed no correlation between the BMD of the fractured femoral head and that of the contralateral hip.

Conclusions: Our results indicate that the BMD of the contralateral hip does not reflect the BMD or the mechanical properties of the fractured femoral head. Therefore, helical blade-type lag screw migration may not be predicted from the BMD of the non-fractured contralateral hip.

\section{Background}

Proximal femoral fractures are one of the most common and devastating consequences of osteoporosis. [1] [2] It is estimated that 1.7 million osteoporotic proximal femoral fractures occur worldwide annually, with mortality rates ranging from $22 \%$ to $29 \%$.[3][4] For their treatment, proximal femoral nails are commonly accepted as an implant of choice. While surgery is mostly successful, failure of lag screws is one of the most severe complications. The typical failure mechanism occurs when the lag screw penetrates or cuts the femoral head; this is termed as "cut through" or "cut out" depending on the type of the lag screw used and on the direction of how the penetration occurs. [5],[6],[7],[8]

A number of hypotheses have been suggested to explain why such a phenomenon occurs and to prevent such failure. While controversial, one of the potential risk factors is poor bone strength.[9][10] As the osteoporotic bone may lack mechanical strength, the bone structure of the femoral head may not provide 
sufficient support, leading to the migration of the lag screw, which may result in penetration of the femoral head. As bone strength is difficult to measure in vivo, the bone mineral density (BMD) is commonly used to quantify and predict the strength of the osteoporotic bone.[11] In cases of a fractured proximal femur, measurements are provided indirectly from the contralateral hip or from the spine. However, there is a paucity of literature on whether indirectly measured BMD from these regions can accurately reflect the mechanical strength of the fractured femoral head. Similarly, it is unclear whether we can predict migration of lag screws from this information.

Therefore, in this study, we performed a biomechanical test on the osteoporotic femoral head to validate whether the indirectly measured BMD from the contralateral hip can elucidate the structural strength of the fractured femoral head and also predict medial migration of lag screws. More specifically, we assessed 1) whether the BMD measured from the contralateral hip can reflect the BMD of the fractured femoral head, 2) whether the BMD of the fractured femur can reflect the mechanical strength of the fractured femoral head, and 3) whether the BMD of the contralateral hip can represent the mechanical strength of the fractured femoral head.

\section{Methods}

The experimental protocol of this study was approved by our institutional review board in priori. The femoral head was retrieved from patients who underwent hip arthroplasty owing to femoral neck fracture between January 2018 and December 2019. The specimens from the patients who agreed to donate the retrieved femoral head for the purpose of this study were selected for analysis. The femoral heads were excluded if acquired from patients 1 ) who had underlying pathologic conditions that may influence bone quality other than osteoporosis, 2) who had undertaken medication that may potentially influence the quality of the bone, 3) whose BMD was not obtained from the contralateral hip owing to the remaining implants from a previous surgery, and 4) with a history of other osteoporotic fractures.

Twenty-four femoral heads that fulfilled our inclusion criteria were retrieved, which constituted the basis of our study. The demographic data of the donors are listed in Table 1.

Table 1. Demographic data of the donor patients

\begin{tabular}{|lll|}
\hline & Mean \pm standard deviation & Minimum/maximum \\
\hline Age (years) & $77.10 \pm 9.92$ & $50 / 94$ \\
\hline Sex (\% of women) & $12(60 \%)$ & \\
\hline Time from fracture to retrieval (days) & $4.74 \pm 3.12$ & $1 / 7$ \\
\hline Body mass index $\left(\mathrm{kg} / \mathrm{m}^{2}\right)$ & $20.89 \pm 2.94$ & $14.47 / 26.23$ \\
\hline
\end{tabular}




\section{BMD measurement of the non-fractured hip}

The BMD of the donor patients was measured from the non-fractured hip using dual-energy X-ray absorptiometry (DXA; Prodigy Advance, GE Healthcare, USA) at the time of admission for surgery. The BMD was measured and collected using the Encore program (GE Lunar Prodigy, USA), with the region of interest (ROI) set at the total proximal femur and femoral neck.

All measurements were performed by two radiotechnologists. Quality control was performed for both technologists and densitometry devices according to the protocol recommended by the International Society for Clinical Densitometry (ISCD). Precision assessment was also performed in priori to measure the least significant difference (LSC), which was $4.2 \%$ in the total hip and $5.1 \%$ in the femoral neck. The measured LSC in our institution is within the recommendation of the ISCD.[12]

\section{Retrieval of the femoral head}

At the time of hip arthroplasty, the femoral head was retrieved with caution to minimize iatrogenic damage. If the ligamentum teres were intact, it was resected with scalpel to prevent avulsion of the femoral head during the dislocation process. The retrieved femoral head was washed with saline and dried at room temperature for $1 \mathrm{~h}$. This was then fresh frozen at $-20^{\circ} \mathrm{C}$ for later experiments.

\section{$B M D$ measurement of the fractured hip}

Direct BMD measurement of the retrieved femoral head was performed using the Quantum GX micro-CT imaging system (PerkinElmer, Hopkinton, MA, USA) within 10 days of the retrieval. The fresh frozen femoral head was thawed at room temperature for $24 \mathrm{~h}$ before the micro-computed tomography (CT) scan. The X-ray source was set to levels of $90 \mathrm{kV}$ and $80 \mu \mathrm{A}$, with a field of view of $72 \mathrm{~mm}$ (voxel size, $144 \mu \mathrm{m}$; scanning time, $4 \mathrm{~min}$ ). Three-dimensional imaging was represented by an existing software within the Quantum GX, and images with a resolution of $4.5 \mu \mathrm{m}$ were obtained for visualization and display. The BMD of the femur was estimated using a hydroxyapatite phantom (QRM-Micro-CT-HA, Quality Assurance in Radiology and Medicine $\mathrm{GmbH}$, Germany), which was scanned using the same parameters.

The spherical cap region of $3 \mathrm{~cm}$ from the fovea capitis was selected as an ROI for the BMD measurement of the retrieved femoral head (Figure 1).

\section{Bone mechanical property test}

Femoral head preparation

Immediately following the BMD measurement with micro-CT, the femoral head was fixed to the custommade jig, which was used as a guide for femoral head resection and fixation during the mechanical test. The jig was manufactured with stainless steel and includes a spherical cap engraving at a depth of 30 $\mathrm{mm}$ to accommodate the femoral head. It includes two holes to insert 2.8-mm K-wires, so that the 
femoral head is firmly fixed within the engraving (Figure 2a). An additional hole was developed at the inferior most region of the engraved sphere to enable penetration of the lag screw during the biomechanical test. A multiple jig with the same design was manufactured with a diameter of engraved hemi-sphere in 4-mm increments from $40 \mathrm{~mm}$ to $56 \mathrm{~mm}$, so that different sizes of the femoral head could be adopted.

The femoral head was fixed in the jig in the position where the region $5 \mathrm{~mm}$ above the fovea capitis is placed at the inferior most part of the sphere cap engraving. The alignment of the femoral head was determined in priori based on the direction of typical lag screw insertion. After placement of the femoral head, the protruded bone out of the jig was resected, so that the resultant femoral head was in the form of a sphere cap of a 30-mm height (Figure 2b).

Lag screw migration test

The mechanical test was performed using a servo-hydraulic universal test machine (MTS Bionix Landmark 370, MTS System Corporation, USA). A helical blade-type lag screw from a commercially available proximal femoral nail system (PFNA-II blade, Depuy Synthes, Switzerland) was utilized to test the mechanical properties of the femoral head. The specifications from the provider indicate that the PFNA-II helical blade has a diameter of $12.2 \mathrm{~mm}$. We used lag screws of $85 \mathrm{~mm}$ in length for the experiment. For the setup, lateral locking of the PFNA-II helical blade was released, so that the blade portion of the PFNA-II lag screw can be freely rotated. This was determined to reproduce the failure mechanism of the lag screw where the femoral head is typically rotated along the lag screw.[13, 14]

Initially, the lag screw was advanced axially along the center of the resected surface of the femoral head until the tip of the lag screw was placed $20 \mathrm{~mm}$ from the outer surface of the femoral head. This was then advanced at $15 \mathrm{~mm} / \mathrm{min}$ until the lag screw penetrated the femoral head and advanced for an additional $5 \mathrm{~mm}$. The load-displacement curve was acquired during the $25-\mathrm{mm}$ advancement of the lag screw. The mechanical strength was defined as the 1) maximum compressive load and 2) accumulated compressive load during the $30-\mathrm{mm}$ advancement. The maximum compressive load was defined as the highest load measured in the load-displacement curve, while the accumulated compressive load was defined as the area under the load-displacement curve during the $25-\mathrm{mm}$ advancement of the lag screw.

\section{Statistical methods}

The measured results were expressed as means and standard deviations. The normality of the distribution of the data was assessed using the Kolmogorov-Smirnov test. Pearson correlation and linear regression analysis were used to determine the correlation between 1) the BMD of the contralateral hip and that of the fractured femoral head, 2) BMD of the fractured femoral head and mechanical properties of the fractured femoral head, and 3) BMD of the contralateral hip and mechanical properties of the fractured femoral head. Statistical analysis was performed using the SPSS software version 20 (SPSS Inc., IL, USA). All P-values were two-sided, and P-values of $<0.05$ were considered significant. 


\section{Results}

The mean interval from the time of injury to DXA was $1.9 \pm 1.1$ days. The mean time from the retrieval of the fractured femoral head to micro-CT and the mechanical test was $5.8 \pm 3.4$ days.

The mean BMD of the contralateral hip measured on DXA was $0.60 \pm 0.14 \mathrm{~g} / \mathrm{cm}^{2}$ in the femoral neck and $0.63 \pm 0.15 \mathrm{~g} / \mathrm{cm}^{2}$ in the total proximal femur, which corresponds to a T-score of $-2.78 \pm 1.19$ and $-3.01 \pm 1.23$, respectively. The BMD of the fractured femoral head measured on micro-CT was $463.3 \pm 48.05$ $\mathrm{mg} \mathrm{HA} / \mathrm{cm}^{3}$. The measured BMDs are summarized in Table 2.

Table 2. Bone mineral density of the fractured femoral head and contralateral hip

\begin{tabular}{|lllc|}
\hline & $\begin{array}{l}\text { Mean } \pm \text { standard } \\
\text { deviation }\end{array}$ & Minimum & Maximum \\
\hline Dual-energy X-ray absorptiometry $\left(\mathrm{g} / \mathrm{cm}^{2}\right)$ & & 0.42 & 0.86 \\
\hline Total hip & $0.63 \pm 0.15$ & 0.41 & 0.83 \\
\hline Femoral neck & $0.60 \pm 0.14$ & & \\
\hline $\begin{array}{l}\text { Micro-computed tomography (mean, } \mathrm{mg} \\
\left.\text { HA } / \mathrm{cm}^{3}\right)\end{array}$ & & & \\
\hline Fractured hip & $463.30 \pm 48.05$ & 345.00 & 583.00 \\
\hline
\end{tabular}

The load-displacement curve during lag screw advancement through the femoral head showed an initial stiff increase followed by a gradual decrease; however, there was a wide variability among the specimens. The mechanical properties as measured by the maximum compressive load and accumulated compressive load are listed in Table 3.

Table 3. Mechanical properties of the fractured femoral head

\begin{tabular}{|llll|}
\hline & Mean \pm standard deviation & Minimum & Maximum \\
\hline Maximum compressive load $(\mathrm{kN})$ & $0.92 \pm 0.35$ & 0.46 & 1.86 \\
\hline Accumulative compressive load $\left(\mathrm{kN}{ }^{*} \mathrm{~mm}\right)$ & $14.50 \pm 5.48$ & 5.48 & 29.76 \\
\hline
\end{tabular}

The correlation among the BMD of the fractured femoral head, BMD of the contralateral non-fractured hip, and mechanical properties of the femoral head is shown in Figure 3. No significant correlation was found between 1) the BMD of the contralateral hip and BMD of the fractured femoral head, 2) BMD and 
mechanical properties of the fractured femoral head, and 3) BMD of the contralateral hip and mechanical properties of the fractured femoral head (Table 4).

Table 4. Correlation between the bone mineral densities and mechanical properties

\begin{tabular}{|llllll|}
\hline & \multicolumn{2}{l}{ Maximum compressive load } & & Accumulated compressive load \\
\cline { 1 - 2 } & Correlation $(r)$ & P-value & Correlation $(r)$ & P-value \\
\hline Contralateral hip & & & & \\
\cline { 1 - 2 } Femoral neck & 0.105 & 0.659 & 0.085 & 0.720 \\
\hline Total hip & 0.142 & 0.551 & 0.092 & 0.701 \\
\hline Fractured hip & 0.317 & 0.173 & 0.230 & 0.330 \\
\hline
\end{tabular}

\section{Discussion}

The results of this study indicate that the BMD of the non-fractured contralateral hip does not reflect the BMD of the fractured femoral head and that this measurement may not be used to predict the medial migration of the lag screw within the fractured femoral head as measured by the axial compression load of the helical blade-type lag screw.

A proximal femoral nail with a helical blade-design lag screw is widely used to stabilize proximal femoral fractures with satisfactory outcomes. $[8,15,16]$ However, a specific type of failure was noted in this implant, termed as "cut in" or "cut through," which is femoral head perforation from a medial migration of the lag screw.[6] This phenomenon has been reported to be observed in $0.7 \%$ to $6.3 \%$ of patients undergoing surgery with a helical blade-type lag screw and is recognized to be one of the fatal complications necessitating subsequent surgery.[17] A number of factors have been suggested to be a potential reason for this phenomenon, which include inappropriate tip-apex distance, unstable fracture pattern, and the z-effect phenomena.[18, 19] In addition, medial migration of the lag screw can also be attributed to the bone strength of the femoral head.[9] Theoretically, the trabecular bone surrounding the helical blade may function to resist further advancement of the lag screw. Therefore, it can be hypothesized that in the femoral head with weak bone strength, resistance may not be sufficient to prevent medial migration.[20][21]

One of the most widely used methods to predict bone strength is measuring the BMD using DXA. However, the BMD largely represents the quantity of the trabecular bone, while bone mass is one of the important factors contributing to bone strength; the mechanical properties of the bone are also configured with other factors, such as architecture geometry, cortical porosity, and tissue mineralization density.[22] Ammann et al. reported that DXA-measured BMD may predict $60 \%$ to $70 \%$ of the variation in 
bone strength according to established studies that validated the correlation between the BMD and bone strength.[23] Regarding the trabecular bone of the proximal femur, Lotz et al. performed a compression test on cylindrical trabecular specimens and compared the results with the BMD measured on quantitative $\mathrm{CT}$. Their analysis showed a high correlation between the bone densities and elastic modulus of the trabecular specimen. However, their study was limited by the small number of specimens because it utilized bone samples from one female and three male cadavers.[24]

Regarding the mechanical properties of the femoral head, there are only a limited number of studies investigating the relationship between the BMD and the mechanical properties of the femoral head. Haba et al. investigated the correlation between the mechanical properties and BMD of 22 femoral heads.[25] They retrieved cylindrical bone samples from the osteoarthritic femoral head and measured the BMD using DXA and quantitative CT. For the mechanical strength test, uniaxial compression tests were performed on the cylindrical trabecular bone to measure the structural modulus and ultimate compression strength. They reported that there was a weak but significant correlation between the BMD and the mechanical properties of the trabecular bone as measured by the uniaxial compression test. In a subsequent study by the same authors, the mechanical properties of the femoral heads from 13 females were measured and compared with the BMD.[26] This time, the BMD was measured before and after femoral head retrieval and after manufacturing of the cylindrical trabecular bone sample. Interestingly, while the BMD measured in the retrieved femoral head showed a significant correlation with both the structural modulus and the ultimate compression strength of the femoral head, as was observed in their previous study, only the structural modulus had a significant correlation with the BMD measured from the femoral neck before retrieval. In our study, the mechanical properties of the femoral head were measured using helical blade lag screw and were presented with the maximum load applied during the compression test and the accumulated load applied during the 25-mm advancement of the lag screw. As we were unable to determine the surface area that directly compressed against the femoral head, we cannot make a head-to-head comparison with the results of other previous studies. However, our findings somewhat contradict other studies in that we found no correlation between the BMD of the femoral head and the mechanical properties as measured by the ultimate and accumulated loads of the helical blade-type lag screw. There are a number of reasons why our study may have showed different results. The most persuasive reason is that a helical blade-type lag screw was utilized to measure the mechanical properties of the femoral head. Because of the helical shape of the blade, the compression load measured in our study should represent the resistance of the trabecular bone compacted against the flanges of the helical blade. However, as rotation of the lag screw was allowed in our study, we think that the flanges of the blade may have cut through the trabecular bone rather than impacting around the flange. This may be the potential reason why the BMD had a limited efficacy in preventing axial migration of the helical blade. Additionally, our cohort comprised patients with osteoporotic fracture; therefore, the bone quantity and quality were considerably lower.

One interesting finding of our study is that there was no correlation between the BMD of the non-fractured hip of the contralateral side and that of the fractured femoral head. A number of studies have reported a correlation of the BMD from the bilateral hip. Banse et al. compared the mechanical properties of 10 
paired left-right proximal femurs.[27] They reported that while a high variation was noted among the bones from different donors, no significant difference was found when both sides were compared. Conversely, a larger study by Afzelius et al. measured the BMD of the bilateral hip in 133 participants and reported that while there was no difference when the BMD of the femoral neck was compared, the total hip BMD was lower in the dominant leg.[28] Li et al. compared the BMD of the non-fractured hip side and fractured hip side using quantitative CT.[29] They reported that the volumetric BMD of the non-fractured side was higher than that of the fractured side and that the difference was significant when the BMD was measured through the center of the femoral neck. In our study, the BMD of the non-fractured side was measured using DXA with the ROI in the femoral neck and total proximal femur. It should be noted that the typical measurement in the total proximal femur does not include the femoral head, as this image overlaps with the acetabular wall. Therefore, our result arises from the comparison among different parts of the proximal femur; this may be one of the reasons why we found no correlation between the BMD of the fractured femoral head and that of the non-fractured contralateral hip.

We acknowledge that there are a number of limitations to our study. First, the study is largely limited by the number and characteristics of the specimens. The retrieved femoral heads utilized in this study were from patients with osteoporotic femoral neck fracture. However, data from different age groups and a wide range of BMD would provide us with a better understanding of the relationship between the BMD and the mechanical strength of the femoral head. Second, our study tested the axial compression load of the helical blade on the femoral head; however, the direction of the applied force does not correspond to the physiologic load applied in vivo. Owing to the anatomical axis of the lower leg, the load against the femoral head should be applied in the cephalomedial direction; however, we were unable to reproduce this in our study.[30] Nevertheless, this is the first study to validate the correlation between the BMD and the mechanical strength of the femoral head as measured by the axial compression load of a helical bladetype lag screw. While the BMD measured from the non-fractured contralateral femoral neck or total proximal femur is the most widely used parameter to predict the bone strength of the fractured femur, our findings suggest that this does not correlate with the BMD or mechanical strength of the fractured femoral head.

\section{Conclusions}

Our study indicates that the BMD of the contralateral hip does not reflect the BMD or mechanical property of the fractured femoral head as measured by the axial compression load using a helical blade-type lag screw. Medial migration of helical blade-type lag screws may not be predicted from the BMD of the nonfractured contralateral hip.

\section{List Of Abbreviations}

BMD, bone mineral density

DXA, dual-energy X-ray absorptiometry 
ROI, region of interest

ISCD, International Society for Clinical Densitometry

LSC, least significant difference

CT, computed tomography

\section{Declarations}

\section{Ethics approval and consent to participate}

The study was approved by the institutional review board of Chosun University Hospital (IRB\#2017-10001). Written informed consent was obtained from all donors of the femoral head in priori.

\section{Consent for publication}

Not applicable.

\section{Availability of data and materials}

The datasets used and/or analyzed during the study are available from the corresponding author upon reasonable request.

\section{Competing interests}

The authors declare that they have no competing interests with regards to this study.

\section{Authors' contributions}

SJ and SAK designed the study and wrote the manuscript. HJL, JHR, BRK, and JHL performed the experiments and analyzed the data. SHL provided essential advice during the writing process. All authors have approved the final version of the manuscript.

\section{Acknowledgments}

We would like to thank Editage (www.editage.co.kr) for English language editing.

\section{References}

1. Kim, B.-S., J.-Y. Lim, and Y.-C. Ha, Recent Epidemiology of Hip Fractures in South Korea. Hip \& Pelvis, 2020. 32(3): p. 119.

2. Yoo, J., et al., Risk factors associated with failure of cephalomedullary nail fixation in the treatment of trochanteric hip fractures. Clinics in Orthopedic Surgery, 2020. 12(1): p. 29-36. 
3. Hurbanova, T., MODERN ASPECTS OF THE PROBLEM OF FRACTURES OF THE PROXIMAL FEMULAR. EUREKA: Health Sciences, 2020(4): p. 88-100.

4. Saad, R.K., et al., Secular Trends of Hip Fractures in Lebanon, 2006 to 2017: Implications for Clinical Practice and Public Health Policy in the Middle East Region. Journal of Bone and Mineral Research, 2020. 35(1): p. 71-80.

5. Zirngibl, B., R. Biber, and H.J. Bail, How to prevent cut-out and cut-through in biaxial proximal femoral nails: is there anything beyond lag screw positioning and tip-apex distance? Int Orthop, 2013. 37(7): p. 1363-8.

6. Frei, H.C., et al., Central head perforation, or "cut through," caused by the helical blade of the proximal femoral nail antirotation. J Orthop Trauma, 2012. 26(8): p. e102-7.

7. Megas, P., et al., Comparative study of the treatment of pertrochanteric fractures-trochanteric gamma nail vs. proximal femoral nail. Zeitschrift fur Orthopadie und ihre Grenzgebiete, 2005. 143(2): p. 252-257.

8. Haynes, R.C., et al., Failure of femoral head fixation: a cadaveric analysis of lag screw cut-out with the gamma locking nail and AO dynamic hip screw. Injury, 1997. 28(5-6): p. 337-341.

9. Bonnaire, F., et al., ["Cutting out" in pertrochanteric fractures-problem of osteoporosis?]. Unfallchirurg, 2007. 110(5): p. 425-32.

10. Bonnaire, F., et al., Treatment strategies for proximal femur fractures in osteoporotic patients. Osteoporosis International, 2005. 16(2): p. S93-S102.

11. Donnelly, E., Methods for assessing bone quality: a review. Clin Orthop Relat Res, 2011. 469(8): p. 2128-38.

12. Schousboe, J.T., et al., Executive summary of the 2013 international society for clinical densitometry position development conference on bone densitometry. Journal of Clinical Densitometry, 2013. 16(4): p. 455-466.

13. Lenich, A., et al., Is the rotation of the femural head a potential initiation for cutting out? A theoretical and experimental approach. BMC musculoskeletal disorders, 2011. 12(1): p. 1-7.

14. Hoffmann, S., et al., Biomechanical evaluation of interlocking lag screw design in intramedullary nailing of unstable pertrochanteric fractures. Journal of orthopaedic trauma, 2013. 27(9): p. 483-490.

15. Sommers, M.B., et al., A laboratory model to evaluate cutout resistance of implants for pertrochanteric fracture fixation. Journal of orthopaedic trauma, 2004. 18(6): p. 361-368.

16. Simmermacher, R., et al., The new proximal femoral nail antirotation (PFNA®) in daily practice: results of a multicentre clinical study. Injury, 2008. 39(8): p. 932-939.

17. Raghuraman, R., J.W. Kam, and D.T.C. Chua, Predictors of failure following fixation of intertrochanteric fractures with proximal femoral nail antirotation. Singapore Med J, 2019. 60(9): p. 463-467.

18. Brunner, A., J.A. Jöckel, and R. Babst, The PFNA proximal femur nail in treatment of unstable proximal femur fractures-3 cases of postoperative perforation of the helical blade into the hip joint. 
Journal of orthopaedic trauma, 2008. 22(10): p. 731-736.

19. Gomes, P.L., et al., Pelvic migration of the helical blade after treatment of transtrochanteric fracture using a proximal femoral nail. Rev Bras Ortop, 2016. 51(4): p. 482-5.

20. Kim, J., K. Choi, and K.H. Yang, New Approach in the Treatment of Intertrochanteric Fracture Using a Cephalomedullary Nail. Journal of the Korean Orthopaedic Association, 2020. 55(3): p. 193-199.

21. Yoo, H., Y. Cho, and S. Hwang, Outcomes of Combined Neck and Trochanter Fractures of the Femur Treated with Cephallomedullary Nail in Elderly. Hip \& Pelvis, 2019. 31(4): p. 200-205.

22. Gong, H., et al., Relationships between femoral strength evaluated by nonlinear finite element analysis and BMD, material distribution and geometric morphology. Ann Biomed Eng, 2012. 40(7): p. 1575-85.

23. Ammann, P. and R. Rizzoli, Bone strength and its determinants. Osteoporos Int, 2003. 14 Suppl 3: p. S13-8.

24. Lotz, J.C., T.N. Gerhart, and W.C.J.J.o.c.a.t. Hayes, Mechanical properties of trabecular bone from the proximal femur: a quantitative CT study. 1990. 14(1): p. 107-114.

25. Haba, Y., et al., Relationship between mechanical properties and bone mineral density of human femoral bone retrieved from patients with osteoarthritis. Open Orthop J, 2012. 6: p. 458-63.

26. Haba, Y., et al., bone mineral densities and mechanical properties of retrieved femoral bone samples in relation to bone mineral densities measured in the respective patients. ScientificWorldJournal, 2012. 2012: p. 242403.

27. Banse, X., et al., Comparative left-right mechanical testing of cancellous bone from normal femoral heads. J Biomech, 1996. 29(10): p. 1247-53.

28. Afzelius, P., M.M. Garding, and S. Molsted, Dual-energy X-ray Absorptiometry of Both Hips Helps Appropriate Diagnosis of Low Bone Mineral Density and Osteoporosis. Diagnostics (Basel), 2017. 7(3).

29. Li, X., et al., The comparison of bone mineral density of femoral head between non-hip fracture side and hip fracture side. Sci Rep, 2020. 10(1): p. 13015.

30. Henschel, J., S. Eberle, and P. Augat, Load distribution between cephalic screws in a dual lag screw trochanteric nail. Journal of orthopaedic surgery and research, 2016. 11(1): p. 41.

\section{Figures}




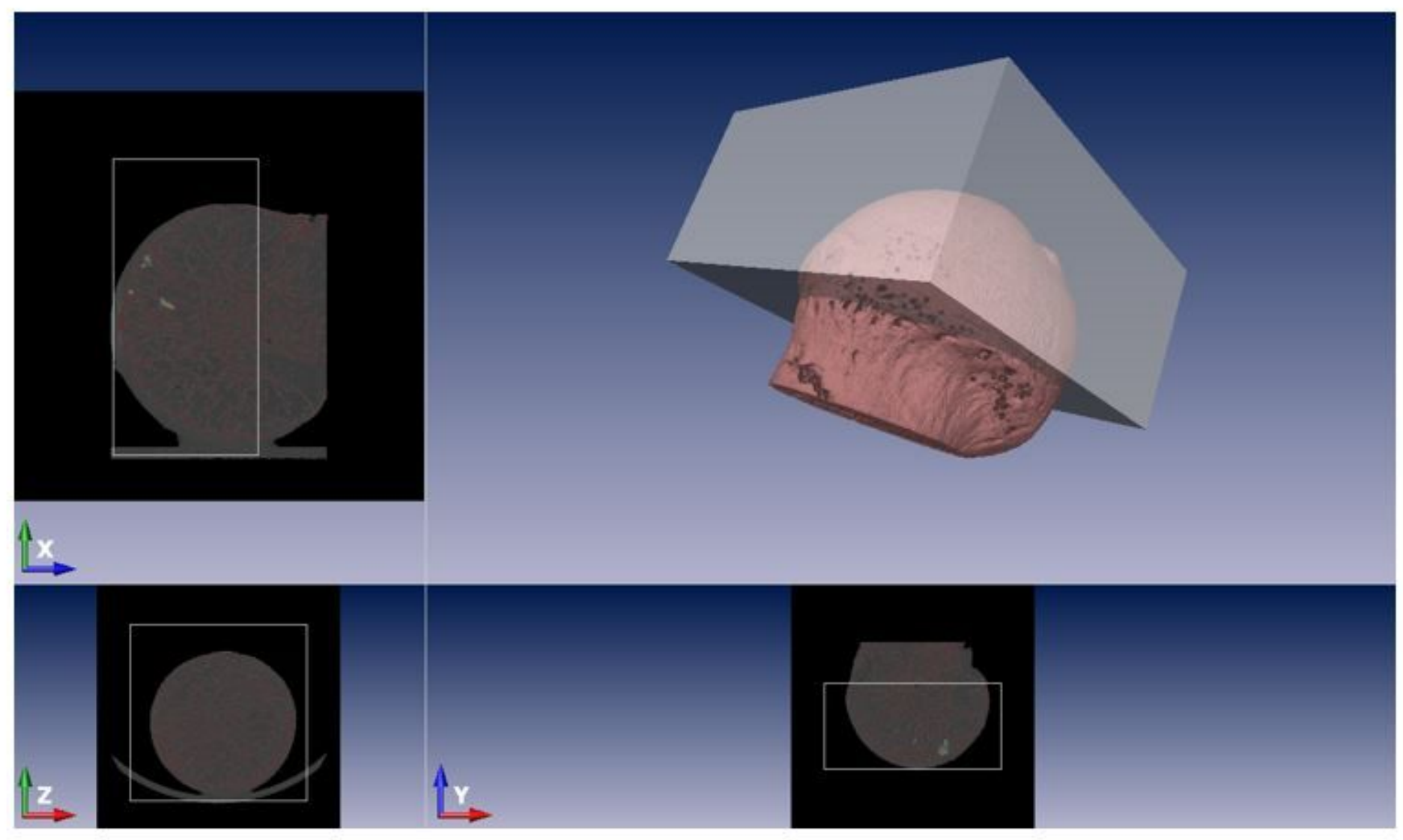

Figure 1

Region of interest for the bone mineral density of the fractured hip using micro-computed tomography 


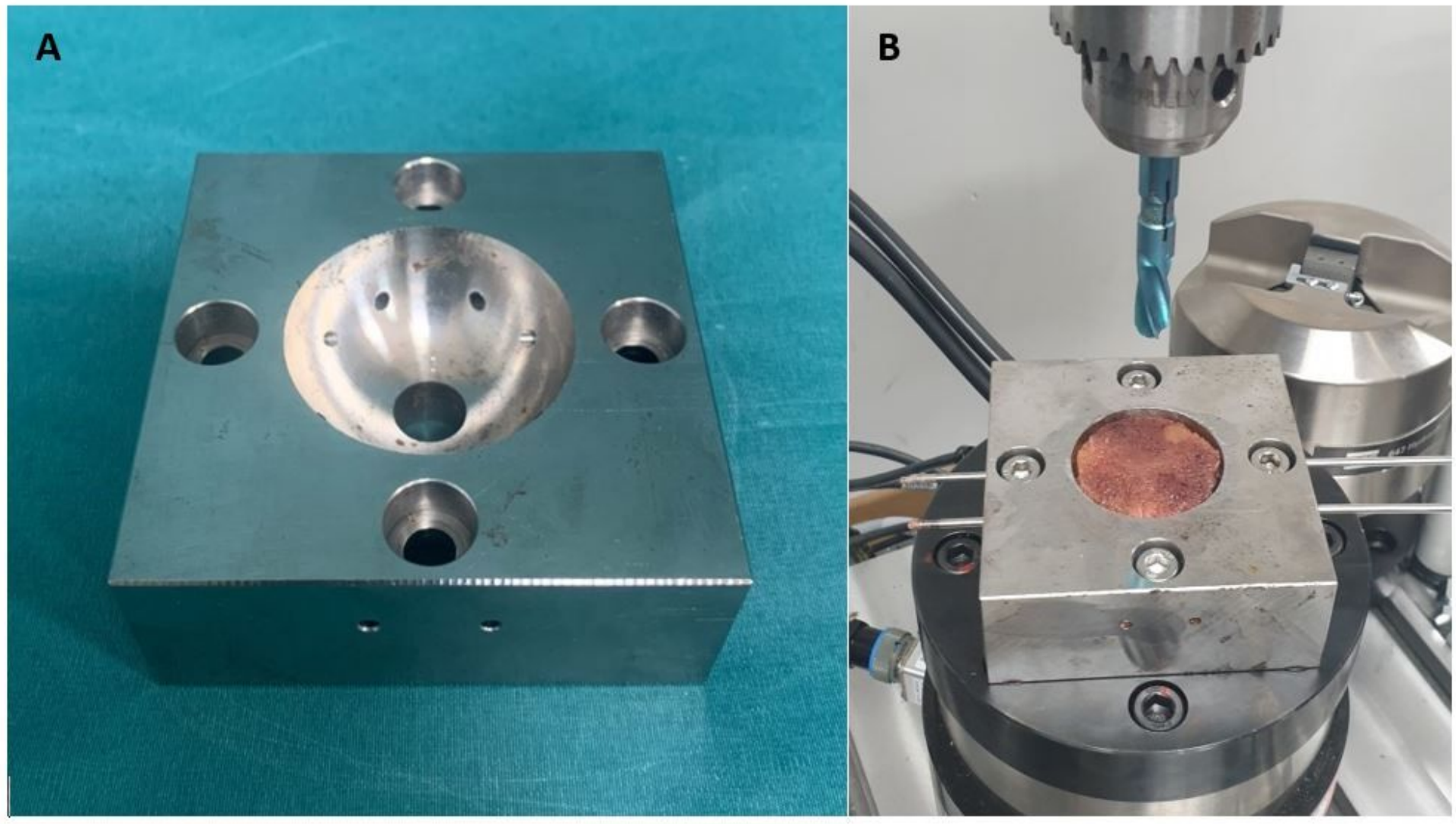

Figure 2

a) Custom-made jig to adopt the retrieved femoral head and b) test setup 

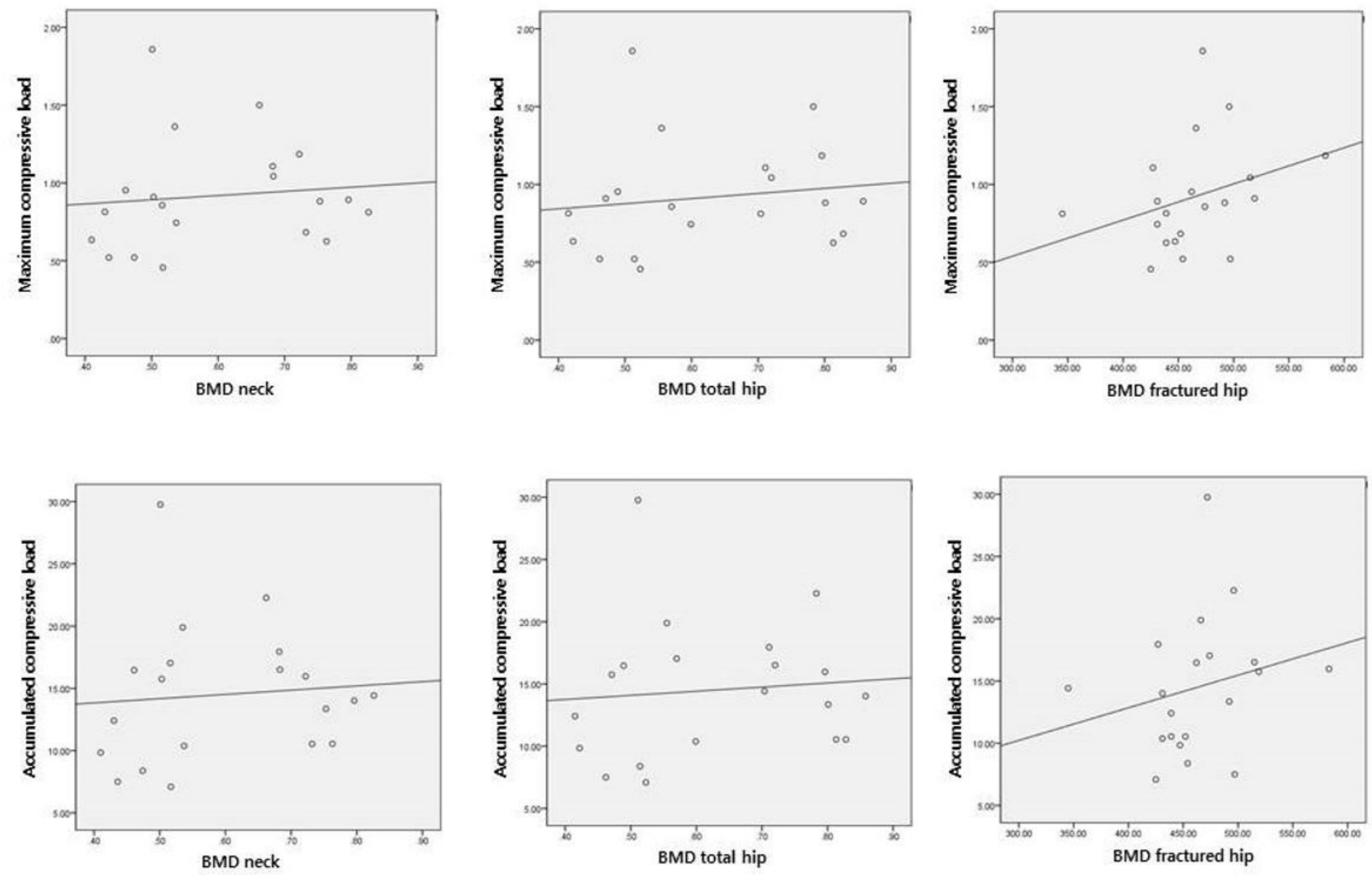

Figure 3

Scattered plot showing the correlation between the BMDs and mechanical properties BMD, bone mineral density 